\title{
The effect of the RadioProtect cosmetic formulation on the skin of oncological patients treated with selected cytostatic drugs and ionizing radiation
}

\author{
Joanna Igielska-Kalwat ${ }^{1}$, Sława Połoczańska-Godek², Dawid Murawa³ , Róża Poźniak-Balicka, \\ Małgorzata Wachowiak', Grzegorz Demski ${ }^{5}$, Sławomir Cieśla ${ }^{4}$ \\ ${ }^{1}$ Symbiosis Dermatology Center, Poznan Science and Technology Park, Poznan, Poland \\ ${ }^{2}$ Higher School of Health, Beauty and Education, Poznan, Poland \\ ${ }^{3}$ Department of Surgery and Oncology, Faculty of Medicine and Health Sciences, University of Zielona Gora, Zielona Gora, Poland \\ ${ }^{4}$ General and Oncological Surgery Clinic, Karol Marcinkowski University Hospital, Zielona Gora, Poland \\ ${ }^{5}$ Aurea Pharma and RadioProtect, Poland
}

Adv Dermatol Allergol 2022; XXXIX (1): 47-51

DOI: https://doi.org/10.5114/ada.2022.113608

\begin{abstract}
Introduction: According to the Polish Central Statistical Office and the National Cancer Registry, cancer is the main cause of death in the world. Every year over 8 million people die from cancer worldwide, while in Poland the disease kills nearly 100,000 people. The group of patients analysed in this study suffered from breast, colorectal and lung cancers, and underwent radio- and chemotherapy. The patients were treated with a cosmetic formulation to accelerate the healing of their skin and improve the functioning of the hydrolipidic barrier.

Aim: To examine oncological patients' skin and to improve its biophysical parameters with a specialized cosmetic preparation.

Material and methods: The study was conducted on 60 female cancer patients treated at the University Hospital in Zielona Gora, Poland. The patients volunteered for in vivo tests of the cosmetic formulation.

Results: The emulsions applied to the patients undergoing chemotherapy significantly increased the hydration of their epidermis. After 4 months of regular use of the preparation, their skin hydration level increased by $22 \%$. The skin hydration of the patients undergoing radiotherapy increased by $3 \%$, as compared with the levels at the beginning of the study.

Conclusions: Cosmetic formulations for oncological patients restore the adequate level of skin hydration, aid the reconstruction of the skin and its protective barrier, soothe irritations, accelerate the healing of pathological lesions and prevent their development. Adverse effects may delay or prevent further treatment. Therefore, appropriately tailored care is very important and should not be overlooked.
\end{abstract}

Key words: cytostatic drugs, ionizing radiation, hydrolipidic barrier, skin hydration.

\section{Introduction}

Oncological therapy is a complex process. Accurate diagnosis is the first and most important stage of therapy for the patient as it allows medical personnel to determine what treatment is necessary and how to plan it. Cancer treatment is now widely recognized as a holistic treatment, where the body is perceived as one connected system. Anti-cancer therapy should not focus on one area of care only, but it should also involve supporting treatment such as proper nutrition, pain management, psychological support, rehabilitation and nursing [1].

Progress in medicine resulted in the development of various cancer treatment methods. One of the most common methods is systemic treatment, which involves systemic drugs. For example, in chemotherapy the patient is treated with cytostatic drugs, which kill cancer cells. These drugs have the longest history as anti-cancer preparations. They have different origins and mechanisms of

Address for correspondence: Joanna Igielska-Kalwat PhD, Symbiosis Dermatology Center, Poznan Science and Technology Park, Poznan, Poland, e-mail: joanna.igielska@wp.pl

Received: 6.05.2020, accepted: 29.08.2020. 
action. Treatment with selected cytostatic drugs mostly consists in targeting rapidly dividing cancer cells. Unfortunately, chemotherapy has side effects and is toxic to healthy cells as well. There are numerous tissues and organs in the human body whose cells are constantly dividing and renewing. Hair follicles and the gastrointestinal mucosa are examples of healthy tissues that renew intensively due to their numerous functions. Apart from cancer cells, it is these cells that get damaged very often. However, ultimately cancer cells are more sensitive to cytostatics because they have more receptors and absorb the substance more quickly. The most common side effects of chemotherapy include hair loss, diarrhoea, fever and leukopenia (decreased number of immune cells), whereas the bacteria and viruses that live in the body become more dangerous for patients undergoing chemotherapy [2-4].

The patients in our study underwent radiotherapy. It is a method of local treatment of tumours with radiant energy by means of devices emitting $\mathrm{X}$-rays or photons. The most common side effects of radiotherapy include overall body weakness, loss of appetite, decreased activity, changes in blood (decrease in white blood cells and platelets), mucous reactions in the mouth, throat, larynx and nasal cavities, respiratory and cardiac reactions, reactions of the intestine, bladder, rectum, vagina, and vulva, radiation-induced skin reactions, and hair loss. In contrast to chemotherapy, the occurrence of side effects depends on the body area exposed to radiation

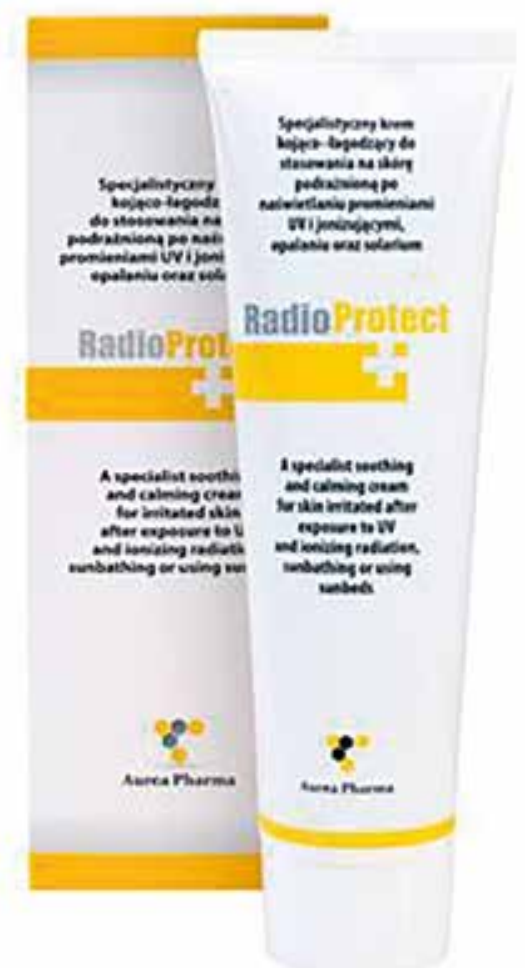

Figure 1. The RadioProtect cosmetic formulation [7] since irradiation works locally only. There are early and late complications and side effects of radiotherapy. Early damage occurs in the tissues whose cells are constantly multiplying. The time of exposure to these complications depends on the lifetime of mature cells. The cells that survived the therapy restore the tissue within 1-2 weeks. Early complications are cured after a short period of time. Late complications of radiotherapy can be observed after a few months or even years. These complications are associated with slowly proliferating tissues, e.g. lungs, kidneys, liver and the nervous system. Complications cause parenchymal cell death or changes in blood vessels, leading to extensive fibrosis, chronic ulceration and necrosis. The risk of late side effects is the main reason for limiting very high doses of radiation $[5,6]$.

\section{Aim}

The aim of the study was to check skin tolerance and the effectiveness of the RadioProtect cosmetic formulation, whose key ingredients were selected to minimize the occurrence of dermatoses as side effects of oncological therapies (chemotherapy and radiotherapy).

\section{Material and methods}

The RadioProtect cosmetic formulation (Aurea Pharma, Poland) was tested on female patients of the University Hospital in Zielona Gora, who underwent chemotherapy due to breast, lung or colorectal cancer. The formulation was applied to eliminate skin reaction to oncological treatment. The patients, who volunteered for the diagnostic and social study, had been treated for cancer between November 2019 and February 2020. Sixty patients met the research criteria and were qualified for tests upon written informed consent. They came mainly from small towns and villages in the Lubuskie Voivodeship. The patients in the study group $(n=60)$ had breast (C50), lung (C34) or colorectal cancer (C18-20). The average age in the study group was 54 (age range: 35-74). The average age in group A was 53 (age range: $37-70$ ), and in group $B-58$ (age range: $35-74$ ).

The patients undergoing radiation therapy were suffering from breast cancer. Before the study the patients' skin was examined in order to diagnose its condition (the zero state). The study was conducted upon the consent of the Bioethics Committee in Poznan. Each participant received a portion of the commercial cosmetic formulation. The patients undergoing chemotherapy applied the emulsion to their faces and necks. Those undergoing radiotherapy applied the cosmetic to the area exposed to radiation, i.e. their breasts. The patients applied the preparation twice a day, in the morning and in the evening. Subsequently, every month digital measurements were taken with Hydro Pen ${ }^{\circledR}$ HP 10 Courage + Khazaka electronic $\mathrm{GmbH}$ hydration measuring device (owned 
by the College of Health, Beauty and Education, Poznan, Poland). The patients were asked to wash their skin with water before applying the cream. The hydration level in superficial skin layers (stratum corneum) was measured with a corneometer. The measurement was based on electrical conductivity - the higher the water content in the epidermis, the lower the reactance, which indicates a higher skin hydration level. The experiment was conducted upon the patients' informed consent. They were also interviewed to gather information on their health and skin condition.

The patients applied the RadioProtect commercial cream (Aurea Pharma, Poland) (Figure 1 [7]) with the following INCI ingredients: Aqua, Olea Europaea Oil, Myristyl Myristate, Maleated Soybean Oil, Cetearyl Olivate, Sorbitan Olivate, Glycerine, Panthenol, Polygonum Cuspidatum Extract, Silybum Marianum Fruit Extract, Squalene, Carbomer, Sodium Hydroxymethylglycinate and Menthol.

In addition, the patients were asked to use their senses of touch, sight and smell to evaluate the sensory characteristics of the cosmetic preparation. Sensory tests of cosmetics are used as the basis for comprehensive assessment of the product characteristics. The results of consumer research are used to assess which properties of the product are particularly important for specific groups of recipients. These tests are based on uniform verification standards, which assess the consumer's feelings when using the product. The following scale was used in the sensory evaluation (Table 1).

\section{Results}

The results of tests on both study groups were compared. Figure 2 shows the average variation in the skin hydration level of the patients who applied the cream during chemotherapy.

After the first month of using the preparation, the patients' skin hydration level increased by $9 \%$. In the subsequent 2 months it increased by $20 \%$, whereas in the fourth month it increased by $21 \%$. The patients' skin hydration level increased linearly over time. After 4 months the parameter increased by $22 \%$.

Figure 3 shows the average variation in the skin hydration level of the patients who applied the cream during radiotherapy.

After the first month of using the emulsion the patients' skin hydration level changed by 1.0\%. The increase in the hydration level was linear until the last week. After 4 months the hydration level increased by 3\%, which indicates that the emulsion was less effective. In conclusion, the emulsion improved the skin hydration level of the patients undergoing radiotherapy, but the product should be used longer to be more effective.

Figure 4 shows the results of evaluation of the patients' satisfaction with the cosmetic. Thirty percent of
Table 1. The assessment scale used for sensory evaluation of the RadioProtect cream (Source: Author's original scale)

\begin{tabular}{lc}
\hline 5 & Very good \\
\hline 4 & Good \\
\hline 3 & Average \\
\hline 2 & Unsatisfactory \\
\hline 1 & Bad \\
\hline
\end{tabular}

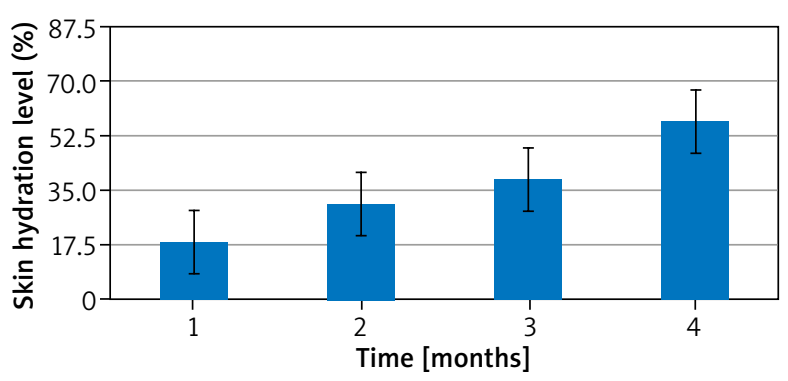

Figure 2. The increase in the patients' skin hydration level after the first application of the formulation and during its use in the course of chemotherapy. ${ }^{* *} p<0.01-$ significant increase from month 1 to month 4 (W4) based on a posthoc test. Test group size $(n=45)$. The skin hydration level was measured once a month

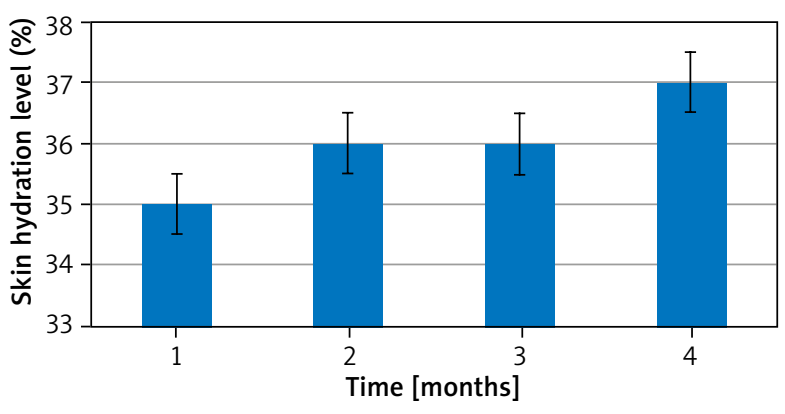

Figure 3. The increase in the patients' skin hydration level after the first application of the formulation and during its use in the course of radiotherapy. ${ }^{* *} p<0.01-$ significant increase from month 1 to month 4 (W4) based on a posthoc test. Test group size $(n=45)$. The skin hydration level was measured once a month

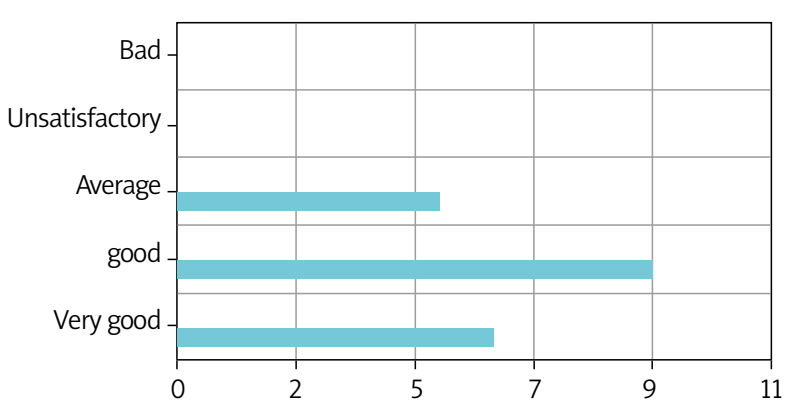

Figure 4. The results of the patients' sensory and hedonic evaluation of the RadioProtect cream 
the patients rated the sensory and hedonic properties of the cosmetic product as very good, $45 \%$ as good, whereas only $5 \%$ rated them as average.

\section{Discussion}

During chemotherapy the skin is dry, hypersensitive, itchy and tends to crack. The hydrolipidic barrier becomes less tight and the body is not sufficiently protected against external factors [3]. Moisturizing preparations should be administered to improve the condition of cancer patients' skin. These preparations do not supply water to the skin, but they limit its loss from the dermis. The most effective method of moisturizing the skin is to strengthen the barriers that inhibit water loss. The reduction of water evaporation from the epidermis is directly related to the increase in skin hydration. The emulsion applied to the patients undergoing chemotherapy significantly increased the epidermis hydration level. It showed that resveratrol, which is the main active ingredient of the emulsion, is a hydrophobe and a very powerful antioxidant. It improves the natural skin repair processes and increases the resistance of skin cells to environmental factors, including UV radiation. The research results suggest that this compound improved skin hydration by stimulating the production of glycosaminoglycans, which are responsible for the proper hydration of deeper skin layers. On the surface of the epidermis they create a filter that is impenetrable for evaporating water. The hydrophobic active substance penetrates the epidermis and thus supplements lipids in the protective layer. In consequence, the emulsion increases the skin hydration parameter $[8,9]$.

Ionizing radiation significantly affects the patient's skin. After only a few sessions it begins to peel, becomes dry and not very elastic, prone to injuries, and abrasions. Long-term patients also suffer from pressure sores. Radiation causes the atrophy of sweat and sebaceous glands and hair in the skin. In order to facilitate skin renewal and accelerate repair processes it is extremely important to take care of it properly, preferably with cosmetics specially prepared for oncological patients. It is crucial to avoid harsh sunlight and to protect elastotic skin with creams with high UV filters. It is necessary to continue this special skincare regime for at least 1 year after the end of therapy. After therapy the skin is also sensitive to cold, since vasospasms caused by a sharp decrease in body temperature lead to extensive ischemia [6]. Our study showed that the emulsion increased the hydration of the stratum corneum in the patients undergoing radiotherapy due to the content of lipid emulsions in its composition. They supplement or modify the natural hydrolipidic layer and intercellular cement. Olive oil inhibits transepidermal water loss (TEWL) by making occlusions and increases hydration of the epidermis by building into cement lipids. It has a nourishing, moisturizing, soothing and regenerating effect on the skin. It is used to treat inflammations. Myristyl myristate and soybean oil also improved the patients' skin hydration levels. They both are greasy emollients creating a protective and nourishing film on the skin surface. The cosmetic also contains squalene, which has antibacterial and antifungal properties. It improves skin lubrication. This compound has film-forming properties, rebuilds the lipid coat of the skin, strengthens it, regulates the activity of sebaceous glands and protects the skin against external factors. Panthenol, which is another component of the cosmetic formulation tested in our study, accelerates healing, soothes irritations, burns and allergies [10-12]. The patients participating in the study applied the cosmetic regularly and reliably. All of them noticed improvement in the hydration, elasticity and condition of their skin in the sensory analysis and all of them found the cosmetic effective. The whole study group agreed that the effects of the product were in line with the manufacturer's declarations.

Like in the study by Bryniarski et al., who observed improvement in the skin hydration level in cancer patients, the application of emollients to the patients in our study also increased this parameter [12]. Morita et al. observed a correlation between the application of individual active substances during chemotherapy and radiotherapy and an increase in skin hydration, improvement of its tightness and function of the hydrolipidic barrier [13]. Nwosu et al. conducted a similar study and found that the skin hydration level in cancer patients was significant as it ranged from 39\% to $74 \%$, depending on the research group [14]. Ratz-Łyko et al. also observed that emollients effectively increased the skin hydration level in patients undergoing chemotherapy and radiotherapy. Depending on the emollient used, the increase in the parameter ranged from $7 \%$ to $29 \%$ [15].

\section{Conclusions}

Cosmetic products for oncological patients restore the adequate level of skin hydration, aid the reconstruction of the skin and its protective barrier, soothe irritations, accelerate the healing of pathological lesions and prevent their development. The emulsion applied in our study may delay the occurrence of adverse effects, which prevent further treatment. Therefore, appropriately tailored care is very important and should not be overlooked.

The study showed that the RadioProtect emulsion improved the condition of the cancer patients' skin, its hydration, and the hydrolipidic barrier. The skin of the patients undergoing radiotherapy regenerated much more slowly. The emulsion should be used for a long period of time to be more effective for patients exposed to ionized radiation.

\section{Conflict of interest}

The authors declare no conflict of interest. 


\section{References}

1. Escudier B, Eisen T, Stadler WM. Sorafenib for treatment of renal cell carcinoma: final efficacy and safety results of the phase III treatment approaches in renal cancer global evaluation trial. J Clin Oncol 2009; 27: 3312-8.

2. Ryan CW, Goldman BH, Lara PN Jr. Sorafenib with interferon alfa-2b in first-line treatment of advanced renal carcinoma: a phase II study of the Southwest Oncology Group. I Clin Oncol 2007; 25: 3296-301.

3. Autier J, Mateus C, Wechsler L. Cutaneous side effects of sorafenib and sunitinib. Ann Dermatol Venereol 2008; 135: 148-53.

4. Berthelet E, Truong PT, Musso K. Preliminary reliability and validity testing of a new Skin Toxicity Assessment Tool (STAT) in breast cancer patients undergoing radiotherapy. Am J Clin Oncol 2004; 27: 626-31.

5. Schmuth M, Sztankay A, Weinlich G. Permeability barrier function of skin exposed to ionizing radiation. Arch Dermatol 2001; 137: 1019-23.

6. Ferrara N, Gerber HP, LeCouter J. The biology of VEGF and its receptors. Nat Med 2003; 9: 669-76.

7. http://www.aureapharma.eu/radio-protect.

8. Yang SC, Tseng CH, Wang PW, et al. Pterostilbene, a methoxylated resveratrol derivative, efficiently eradicates planktonic, biofilm, and intracellular MRSA by topical application. Front Microbiol 2017; 8: 1103-10.

9. Yang T, Wang L, Zhu M, et al. Properties and molecular mechanisms of resveratrol: a review. Pharmazie 2015; 70: 501-6.

10. Lacouture ME, Maitland ML, Sagaert S. A proposed EGFR inhibitor dermatologic adverse event-specific trading scale from the MAASCC skin toxicity study group. Sup Care Cancer 2010; 18: 509-22.

11. Autier J, Escudier B, Wechsler J. Prospective study of the cutaneous adverse effects of sorafenib, a novel multikinase inhibitor. Arch Dermatol 2008; 144: 886-92.

12. Bryniarski P, Andrysiak A, Jezioro M, et al. Evaluation of prognostic factors, symptoms and consequences of dehydration in patients with cancer based on retrospective data analysis of 102 patients treated in the Department of Palliative Medicine at the University Hospital in Cracow - preliminary report. Foll Medica Cracoviensia 2017; 57: 5-14.

13. Morita T, Hyodo I, Toshimi T, et al. Association between hydration volume and symptoms in terminally ill cancer patients with abdominal malignancies. Ann Oncol 2005; 16: 455-60.

14. Nwosu AC, Mayland CR, Mason S, et al. The association of hydration status with physical signs, symptoms and survival in advanced cancer - the use of Bioelectrical Impedance Vector Analysis (BIVA) technology to evaluate fluid volume in palliative care: an observational study. Liver Dis 2016; 11: e0163114.

15. Ratz-Łyko A, Arct J, Pytkowska K. Moisturizing and anti-inflammatory properties of cosmetic formulations containing Cantella asatica extract. Indian J Pharm Sci 2016; 78: 27-33. 